\title{
ECOLOGIA DEL COTO MONO (Alouatta seniculus, CEBIDAE) EN EL RIO PACAYA, RESERVA NACIONAL PACAYA-SAMIRIA, PERU
}

Pekka Soini*

\section{RESUMEN}

Desde 1979 hasta 1992 se investigó la ecología del coto, Alouatta seniculus en el río Pacaya medio, con énfasis en los aspectos de densidad poblacional, organización social, reproducción, uso de hábitat y alimentación. La población de cotos se compone de grupos heterosexuales, grupos formados únicamente de machos y animales solitarios. Los grupos heterosexuales se componen de 2 a 11 (excepcional mente hasta 13) integrantes (promedio $=5.54$ ). Cada grupo tiene generalmente $1-2$ (promedio $=1.3$ ) machos adultos y 1-3 (promedio $=1.8$ ) hembras adultas. Los adultos constituían aproximadamente el $60 \%$ de la población total. La densidad poblacional fue 36 animales/ $\mathrm{km} 2$. En la Reserva Nacional Pacaya Sumiría el coto es el mamífero arbóreo de mayor biomasa (162 kg/km2 en el área del estudio). Los nacimientos ocurren durante todo el año, pero con mayor frecuencia en octubre-abril y son muy escasos en julio-setiembre. Los intervalos observados entre nacimientos sucesivos fluctúan de 15 a 28 meses. Las crías son cargadas hasta 5-6 meses de edad y únicamente por las hembras. Los cotos ocupan principalmente los estratos superiores del bosque, pero en marzo mayo frecuentan también el estrato inferior, El coto fue el único primate que se ha observado cruzar voluntariamente a nado el río, por lo que éstos no constituyen una barrera mayor de dispersión para esta especie. L as áreas domiciliarias de grupos contiguos se sobreponen parcialmente; para 2 grupos éstas tenían una extensión aproximada de 9 ha y 6 ha, respectivamente. El coto se alimenta principalmente de hojas e yemas ( $53 \%$ de la dieta anual), frutos $(40 \%)$ y flores $(6 \%)$, pero también de pecíolos, ramitas tiernas y brotes terminales de lianas. La composición proporcional de la dieta fluctúa estacionalmente, siendo los frutos el recurso más importante $(64.7 \%)$ en la estación medio de lluvia (enero-marzo) y las hojas y yemas en las otras estaciones.

Instituto de Investigaciones de la Amazonía Peruana (IIAP). Av. A belardo Quiñónez. Km. 2.5. A partado 784. Iquitos - Perú. 
Palabras Claves: Alouatta seniculus, ecología, dinámica poblacional, reproducción, dieta, densidad poblacional, Reserva Nacional Pacaya-Sumiría, Perú.

\section{ABST RACT}

Population density, social organization, reproduction, habitat use, and diet of the red howler monkey, Alouatta seniculus was studied in the Pacaya river basin, A mazonian Peru from 1979 to 1992. The study population contained heterosexual and all male groups, and solitary animals. The heterosexual groups had 2-1 1 (exceptionally up to 13$)$ members $(x=5.54)$, generally $1-2(x=1.3)$ adult males and $1-3 x=1.8$ ) adult females. A dults comprised about $60 \%$ of the total population. Ecological population density was 36 animals $/ \mathrm{km} 2$. In the Pacaya-Samiria N ational Reserva $A$. seniculus attains a higher biomass than any other arboreal mammal (162 kg/km2 in the study area). Birth occured yearround, but they were most frequent in October-A pril and very infrequent in JulySeptember. The observed interbirth intervals ranged from 15-28 months. Infants were carried until 5-6 months oid, apparently only by adult females. A. seniculus occupied mainly the upper forest strata, but in $M$ arch-M ay they also frequented subcanopy strata. It was the only primate seen vountary swimming across the river; these do not seem to constitute dispersal barriers for $A$. seniculus. The borne ranges of contiguos groups over lapped partially. The borne ranges of two groups measured $9 \mathrm{Ha}$ and $6 \mathrm{Ha}$, respectively $\mathrm{A}$. seniculus feeds mainly on leaves and leaf buds (53\% of annual diet), fruits (40\%), and flowers $(6 \%)$, hut also on petioles, young stems, and terminal shoots of vines. Composition of the diet showed seasonal changes, fruits heing the most important resource $(64.7 \%)$ in mid wet-season ( anuary-M arch) and leaves in the other seasons.

\section{INTRODUCCION}

El coto mono, Alouatta seniculus es uno de los primates más grandes de Ia A mazonía; un macho adulto y una hembra adulta, cazados por un vigilante en el río Pacaya el 30.03 .82 , pesaron $7.7 \mathrm{~kg}$ y $4.9 \mathrm{~kg}$. Respectivamente. Debido a su tamaño y la facilidad de su caza es muy perseguido para el consumo por los habitantes de la región, y corno consecuencia de ello ha sido exterminado 0 reducido a niveles poblacionales extremadamente bajos en muchas áreas de la región amazónica (Freese et aL, 1982; Soini, 1982). En la Reserva Nacional Pacaya-Sumiría es abundante, y desde 1979 se ha estado realizando investigaciones de la ecología y dinámica poblacional del coto y de las otras especies de primates presentes en la Reserva. 
Los objetivos planteados para la presente investigación estuvieron referidos a determinar importantes parámetros poblacionales, como la densidad de la población, composición por edad y sexo, nacimientos; asimismo se determinó el régimen alimenticio y el área domiciliaria. En el presente informe se reportan los resultados obtenidos de las investigaciones de la especie Alouatta seniculus. 


\section{AREA DE ESTUDIO}

Las observaciones de campo fueron realizadas principalmente en los alrededores de la Estación Biológica de Cahuana, sito en el río Pacaya medio, en un área total de aproximadamente $11 \mathrm{~km} 2$. En ésta extensión, se definió como área principal de estudio la isla Cahuana y la zona colindante con la tipishca (antiguo meandro del río) del mismo nombre (Figura 1). Excluyendo los cuerpos de agua y los pantanos abiertos ("pungales"), la extensión total del área boscosa es $7.2 \mathrm{kni} 2$, de los cuales $4 \mathrm{~km} 2$ corresponden a la superficie boscoza de la isla Cahuana.

El área de estudio consiste en suelos aluviales, bajos y planos, sujetos a inundación anual. El patrón climatológico anual comprende una estación lluviosa larga, desde octubre hasta junio, y una estación relativamente seca, desde junio hasta comienzos o mediados de octubre (Figura 2). El río Pacaya comienza a aumentar su caudal en octubre 0 noviembre y desde febrero hasta mayo 0 junio casi todos los terrenos del área de estudio permanecen inundados.

Fenológicamente, la estación lluviosa se caracteriza por una producción abundante de frutos, los que empiezan a escasear hacia fines de la estación y se hacen notoriamente escasos en la estación seca y hasta el comienzo de la lluviosa.

\section{METODOS}

Las observaciones del coto en el Pacaya medio se iniciaron en 1979 y fueron continuadas periódicamente hasta el presente, pero en forma más intensiva y sostenida en los 3 últimos años (1989-91). Estas observaciones abarcan todos los meses del año en horas del día.

Para determinar la densidad poblacional se recorrió inter-diariamente las trochas previamente abiertas en el interior de la isla, señaladas cada $100 \mathrm{~m}$ y graficadas en un mapa matriz, y por la cocha se recorrió por las orillas de la isla en canoa a remo. Al encontrar un grupo de cotos, se anotó su ubicación y se procuró averiguar su tamaño y composición completa. Para lograr esto, se hizo necesario observar el grupo por varias horas e inclusive días. La ubicación de cada grupo encontrado (o reencontrado) fue luego ploteado en el mapa matriz.

Para determinar la composición de los grupos se estableció 5 categorías de edad, basadas en el tamaño, aspecto y comportamiento del animal, como sigue:

Infante : Cría pequeña, cargada constante 0 intermitentemente por la madre; edad 0 - 5 meses. 
Juvenil 1 : Todavía de tamaño muy pequeño, de locomoción propia (pero puede ser cargado brevemente en situaciones de peligro); se asocia constantemente con su madre.

Juvenil 2 : Conspicuamente mayor que las crías dependientes, la mitad 0 un poco más, del tamaño adulto; aunque todavía se asocia frecuentemente con su madre anda muy independiente de ella.

Subadulto : Tamaño casi adulto, pero perceptiblemente menos corpulento que éste.

Adulto : Tamaño y forma adulta, macho con región hioidea muy desarrollada; hembra a menudo acompañada de un infante y/o juvenil.

El color claro y la forma pendulosa del escroto permitió identificar a los machos adultos y subadultos sin ninguna duda desde cierta distancia, pero la identificación del sexo de los juveniles fue más difícil y ambigua e imposible de los infantes. Debido a que los grupos sociales de esta especie son muy conspicuos y sedentarios y mantienen generalmente una composición bastante estable, la mayoría de los grupos presentes en el área pudieron ser reconocidos individualmente. Esto permitía un monitoreo general de la aparición de nuevas crías en los grupos y, en muchos casos, conocer la fecha o mes de nacimiento de éstas, y por ende llevar un registro acumulativo mensual de nacimientos observados en la población.

Para conocer la dieta alimentaría del coto, se anotó cada vez que se observó un grupo 0 individuo alimentarse de una planta, o conjunto de plantas de la misma especie, la identidad taxonómica de la planta y la parte consumida (hojas, frutos, flores, brotes, tallos, etc.); si fue luego visto comer en otro lugar se anotó esto como otra observación, y así sucesivamente. En esta forma se obtuvo un registro acumulado y cuantitativo de observaciones de alimentación. También se recogió muestras de herbario de la mayoría de especies de plantas consumidas por el coto, las que fueron posteriormente identificadas taxonómica mente por los botánicos Dr. F anklin A yala y Dr. Sydney M CD aniels.

Para indagar sobre la utilización del espacio, se monitoreó intensivamente los movimientos de 2 grupos contiguos periódicamente, por un período mayor de un año. El área total utilizada por cada grupo fue estimada calculando la extensión total del área encerrada por el polígono que une los puntos periféricos de la distribución de todas las ubicaciones registradas para el grupo durante el monitoreo. 


\section{RESULTADOS Y DISCUSION}

\section{Tamaño y composición de los grupos sociales}

En el Pacaya el coto vive mayormente en grupos heterosexuales de 2 a 11 animales incluyendo las crías dependientes, siendo el tamaño modal de los grupos 5 animales y el tamaño promedio $5.54+<1.59$ D.E.; $(n=74)$. Se observó en 3 oportunidades grupos que tenían temporariamente 13 miembros, pero los grupos

mayores de 11 miembros parecen ser raros y de composición inestable, 0 representan coaliciones temporarias de 2 grupos distintos.

También se observa en la población, algunos grupos conformados únicamente por 2 ó 3 machos adultos y/o subadultos. Asimismo la presencia de animales solitarios es muy común en esta especie. Mayormente son machos adultos y subadultos, pero en 3 oportunidades se ha encontrado hembras solitarias.

L os grupos heterosexuales contienen generalmente 1 ó 2 machos adultos y ocasionalmente hasta 3. A próximadamente el $55 \%$ de los grupos tienen sólo un macho adulto y el 45\% 2 ó 3. La mayoría de los grupos contienen 2 ó 3 hembras adultas, pero apróximadamente el $23 \%$ de los grupos tienen sólo una. En término medio el número de hembras adultas por grupo es mayor que el de machos adultos (1.8 versus 1.3). El número de animales inmaduros por grupo varía generalmente de 0 a 5. La composición típica o modal de los grupos es: 1 machos adulto +2 hembras adultas +2 animales inmaduros. A próximadamente el $60 \%$ de la población de estudio consistía en animales adultos y el $40 \%$ en animales inmaduros.

La Tabla 1 presenta la composición por edad y sexo de los 16 grupos Estudiados en Cahuana. La composición porcentual por edad y sexo es la siguiente:

$M$ achos adultos

Hembras adultas

M achos subadultos

Hembras subadultas

Juveniles (ambos sexos)

Infantes (ambos sexos)
$26 \%$

$34 \%$

$7 \%$

$2 \%$

$21 \%$

$10 \%$

La mayor proporción de hembras adultas que machos adultos y la menor proporción de hembras subadultas parece deberse, por lo menos en parte, a que las hembras de esta especie alcanzan la adultez en una edad más temprana que los machos (Crockett y Eisenberg, 1987). 


\section{Densidad poblacional}

El censo de la población total de cotos de la isla de Cahuana (Figura 1) indicó que estaba habitada por 24 grupos heterosexuales, con un total aproximado de 137 animales. Además de estos grupos estables, la población incluía unos 3 animales solitarios y 2 parejas incipientes, por lo que la población total consistía en aproximadamente 144 animales. Considerando que la isla tiene una extensión total de $4.3 \mathrm{~km} 2$, de la cual aproximadamente $0.3 \mathrm{~km} 2$, es pantano abierto (pungales) y $4.0 \mathrm{~km} 2$ bosque, podemos concluir que la densidad ecológica del coto en la isla es 36 animales $/ \mathrm{km} 2$. Los censos menos detallados realizados en los otros sectores del área de estudio reportan una

densidad similar que lo hallado en la isla, por lo que consideramos 36 cotos $/ \mathrm{km} 2$ como representativo para todo el Pacaya medio.

Si consideramos $6 \mathrm{~kg}$ como peso promedio de los adultos de A. seniculus y el $75 \%$ de éste como peso promedio para todas las categorías de edad (Freese et al., 1982), podemos estimar la biomasa del coto en el Pacaya medio en $162 \mathrm{~kg} / \mathrm{km} 2$.

Una comparación con las densidades ecológicas y biomasas de las otras 6 especies de primates diurnos presentes en la isla (Tabla 2) demuestra que el coto es la seguna especie más abundante en términos de número de animales y el más abundante en t\& minos de biomasa. En la Reserva Pacaya Samiría el coto es el mamífero arbóreo de mayor biomasa.

En 1974 F reese y colaboradores realizaron censos generales de primates en 9 áreas principales de la selva baja (F reese et at., 1982), obteniendo estimados de densidades poblacionales del coto sólo para 2 de estas áreas; en las 7 áreas restantes la especie había sido exterminada o reducida a niveles poblacionales tan bajas por la caza excesiva, que no encontraron ejemplar alguno en los recorridos de censo. Las únicas dos áreas fueron el río Samiria, en la Reserva $\mathrm{N}$ acional Pacaya-Samiria, y cocha Cashú, en el Parque Nacional Mamá. En la primera obtuvieron una densidad de 30.5 animales $/ \mathrm{km}$ y en la segunda 24.0 animales/ $\mathrm{km} 2$, censos posteriores y más intesivos de Terborgh (1983) en esta última área dieron una densidad de 30 animales/km2. Por lo tanto, la densidad obtenida en el presente estudio indica que la abundancia del coto en el Pacaya medio es similar o algo mayor que en las citadas áreas. Indudablemente la población más extensa y abundante del país se encuentra en la Reserva $\mathrm{N}$ acional Pacaya-Samiria. 


\section{Reproducción}

La hembra de A. seniculus alcanza la madurez sexual entre los 35 y 4.5 años de edad (Crockett y Rudran, en Crockett y Eisenherg, 1987); es poliestral y experimenta ciclos de estro durante todo el alio. El ciclo de estro tiene una duración de 17 días y la gestación 186-194 (promedio = 191) días (Crockett y Seculic, 1982).

En el período 1979-9 1 obtuvimos 49 registros de nacimientos con las fechas 0 meses de nacimiento conocidas. Estos indican que los nacimientos ocurren durante todo el alio (Figura 3). La frecuencia de nacimientos no parece ser uniforme a través de las estaciones: se observa en la población el mayor número de neonatos y crías pequeñas en el período octubre - abril y en el período julio - setiembre se ven muy pocos. Este último período corresponde a la estación seca del alio y la escasa presencia de neonatos y crías muy pequeñas probablemente indica una reducción de nacimientos en ese período; también podría deberse en parte a una mayor mortalidad perinatal entre las crías que nacen entonces.

Una hembra adulta individualmente reconocible, cuya trayectoria se siguió por más de 9 años (1981-90), tuvo 4 crías en ese período. Los intervalos entre 3 sucesivos nacimientos, con las fechas exactas 0 aproximadas conocidas, fueron 15 meses y 18 meses, respectivamente. En otra hembra reconocible, que seguimos por más de 3 años (1987-9 1), el intervalo entre los nacimientos de sus 2 crías fue 27628 meses. En los casos que se citan la hembra estaba al momento de alumbrar todavía acompañada por su

cría anterior, por lo que esos intervalos probablemente representan casos normales. Cuando la hembra pierde su cría a temprana edad, el intervalo hasta el siguiente alumbramiento puede ser considerablemente más corto.

\section{Cuidado y desarrollo de las crías.}

Durante el primer mes de vida la madre carga la cría sobre el pecho y vientre; en el transcurso del segundo mes pasa a ser cargada sobre la espalda. En esta especie las crías son cargadas únicamente por las hembras; hemos podido constatar que ni siquiera en situaciones de extrema alarma las crías suben en la espalda de algún macho. Sin embargo, en algunas oportunidades vimos machos proteger crías interponiéndose entre la cría amenazada y el origen del peligro.

En el cuarto mes las crías empiezan a movilizarse por sí solas, alejándose varios metros de sus madres, pero siguen siendo cargadas frecuentemente hasta los 5 meses de edad; en el sexto mes son cargadas sólo en situaciones amenazantes. 


\section{Preferencias y uso de hábitat}

El coto habita todos los tipos de bosque existentes en el Pacaya medio; pero en y alrededor de los bosques de restinga (Encarnación, 1985) la densidad de grupos de cotos tiende a ser algo mayor que en los bosques pantanosos o de bajial.

El coto ocupa principalmente el estrato superior del bosque. En marzo, abril y mayo pasa también mucho tiempo alimentándose en niveles muy bajos de la vegetación, a menudo en alturas de sólo 1 - 3 ni sobre el suelo o superficie del agua; en octubre a febrero se le observa sólo raras veces en ise nivel de la vegetación y nunca en julio, agosto y setiembre.

Normalmente los cotos nunca bajan al suelo, pero en una oportunidad se observó a una hembra hacerlo para recoger su cría aparentemente muy debilitada, que había caído al suelo; en otra oportunidad, a un macho saltar al suelo y correr por él una distancia considerable, al percatarse de la presencia humana.

En una oportunidad se observó un macho dispersante meterse deliberadamente al agua y cruzar a nado el Río Pacaya, que allí tenía un ancho de $80 \mathrm{ni}$. El coto es la única especie de mono que se observó cruzar voluntariamente tules cuerpos mayores de agua, lo que indica que los ríos no constituyen una barrera para la dispersión de esta especie.

En comparación con otros primates mayores, el coto es bastante sedentario. Los dos grupos mejor estudiados en este aspecto ocupaban áreas domiciliarias de 9 ha y 6 ha de extensión, respectivamente (Figura 4). Los grupos heterosexuales defienden las áreas que ocupan mediante coros de aullidos. No obstante, las áreas domiciliaras de grupos contiguos se traslapan ampliamente (Figura 4).

Los cotos pasan la noche sobre las ramas mayores de árboles emergentes 0 del estrato superior del dosel. Todo el grupo duerme generalmente en la copa del mismo árbol, acurrucados en un solo grupo o en 2 a 4 subgrupos repartidos en diferentes partes de la copa. Generalmente un grupo tiene varios árboles preferidos de dormir, pero puede pasar varias noches consecutivas en el mismo árbol.

\section{Alimentación}

La dieta del coto en el Pacaya consiste principalmente de hojas, yemas, frutos, flores, pecíolos, brotes terminales de lianas y ramitas tiernas. Los cotos parecen ser exclusivamente vegetarianos, ya que nunca los observamos alimentarse de recursos de origen animal. La población en estudio se observó alimentarse de más de 40 especies de árboles, lianas y hemiepífitas. La Tabla 3 presenta una 
lista taxonómica de las especies de plantas identificadas que le sirvieron de alimento.

En 412 registros de alimentación las frecuencias porcentuales de consumo de los diferentes componentes de la dieta fueron hojas $53 \%$, frutos $40 \%$, flores $6 \%$ y otros 1\%. Las hojas de árboles y lianas fueron las más importantes; y aunque comían también hojas maduras, más del $90 \%$ eran yemas y hojas tiernas. De algunas especies de árboles comían sólo la parte distal de las hojas (por ej. Ceiba pentandra, Couropita amazónica e Inga sp.). Las fuentes cuantitativamente más importantes de hojas fueron los árboles Cecropia membranacea, Clarisia biflora, Pithecolobium muliflorum e Inga spp.

Los frutos siguieron en importancia y además de los maduros, consumían con mucha frecuencia también frutos inmaduros. Los de mayor consumo fueron Ficus spp. Clarisia biflora, Cecropia membranacea, Inga spp, Brosimum rubescens, y Xylopia ligustrifolia (sólo en enero - marzo).

El consumo de flores incluía unas 10 especies, de las cuales ninguna se destacaba en importancia. El consumo de pecíolos, brotes terminales de lianas y ramitas tiernas era relativamente infrecuente y contribuía muy poco a la dieta total del coto. Las proporciones de los diferentes componentes vegetales en la dieta variaban entre las estaciones anuales (Tabla 4). Las hojas constituían el mayor recurso durante toda la estación seca y el trimestre inicial de la estación Iluviosa; en el trimestre medio de la estación lluviosa los frutos fueron el recurso principal y en el trimestre final de la estación lluviosa los cotos se alimentaban de hojas y de frutos con casi igual frecuencia (Tabla 4). Las flores fueron un recurso poco importante, excepto en el trimestre final de la estación lluviosa, cuando casi el $9 \%$ de los registros de alimentación correspondían al consumo de flores.

\section{AGRADECIMIENTOS}

Los estudios de primates y otra fauna en la Reserva Nacional PacayaSamiria formaron parte de las actividades del programa de desarrollo integral de la Reserva, del Ministerio de A gricultura y, posteriormente, del Comité Local de Desarrollo de la Reserva Nacional Pacaya-Samiria (COREPASA), Iquitos. En la fase inicial estas actividades recibieron apoyo financiero del Gobierno Suizo, a través del programa "Cooperación Técnica Suiza", y posteriormente de World Wildlife Fund (WWF - U.S.). La fase final (199 1-1992) del estudio se realizó dentro del programa de investigación de fauna del Instituto de Investigaciones de la Amazonía Peruana(IIAP).Las identificaciones de las muestras botánicas recolectadas para este estudio fueron realizadas por el Dr. Franklin Ayala (Universidad Nacional de la Amazonia Peruana, Iquitos) y el Dr. Sydney M cDaniel (Instituto for Botanical Explotarion, M ississippi, U.S.A.). M aría Soini participó ampliamente en las actividades de campo, brindando un apoyo 
indispensable. Ofrecemos nuestro reconocimiento y agradecimiento a todas estas personas e instituciones por su valiosa colaboración.

\section{BIBLIOGRAFIA}

Crockett, C.M .y J.F. Eisenberg, 1987. Howlers: V ariations in group size and demography. Pp. 54-68, en Primate Societies (editado por Smuts, B.B.

et

al.). Univ. Chicago Press, Chicago.

Crockett, C.M y R. Seculic, 1982. Gestation length in red howler monkeys. Am. J. Primatol., 3: 291-294.

Encamación, F., 1985. Introducción a la llora y vegetación de la A mazonía Peruana: Estado actual de los estudios, medio natural y ensayo de una clave de determinación de las formaciones vegetales en la llanura amazónica. Candollea, 40: 237-252.

Freese, C.H.; P.G. Heltne; N. Castro y G. Whitesides, 1982. Pattems and determinants of monkey densities in Peru and Bolivia, with notes on distributions. mt. 1. Primatol., 3: 53-90.

Soini, P., 1982. Primate conservation in Peruvian A mazonia. mt. Zoo Year Book, 22:37-47.

Soini, P., 1986. A synecological study of a primate community in the Pacaya Sainiria National Reserve, Peru. Primate Conservation, (7):63-71.

Terborgh, J. 1983. Five New World Primates. Princeton Univ. Press., Princeton, $260 \mathrm{pp}$. 
TABLA 1. Composiciones anuales - por edad y sexo de 16 grupos heterosexuales de Alouatta seniculus por edades en Cahuana, Río Pacaya.

Grupo Adultos Subadultos Juveniles Infantes Total

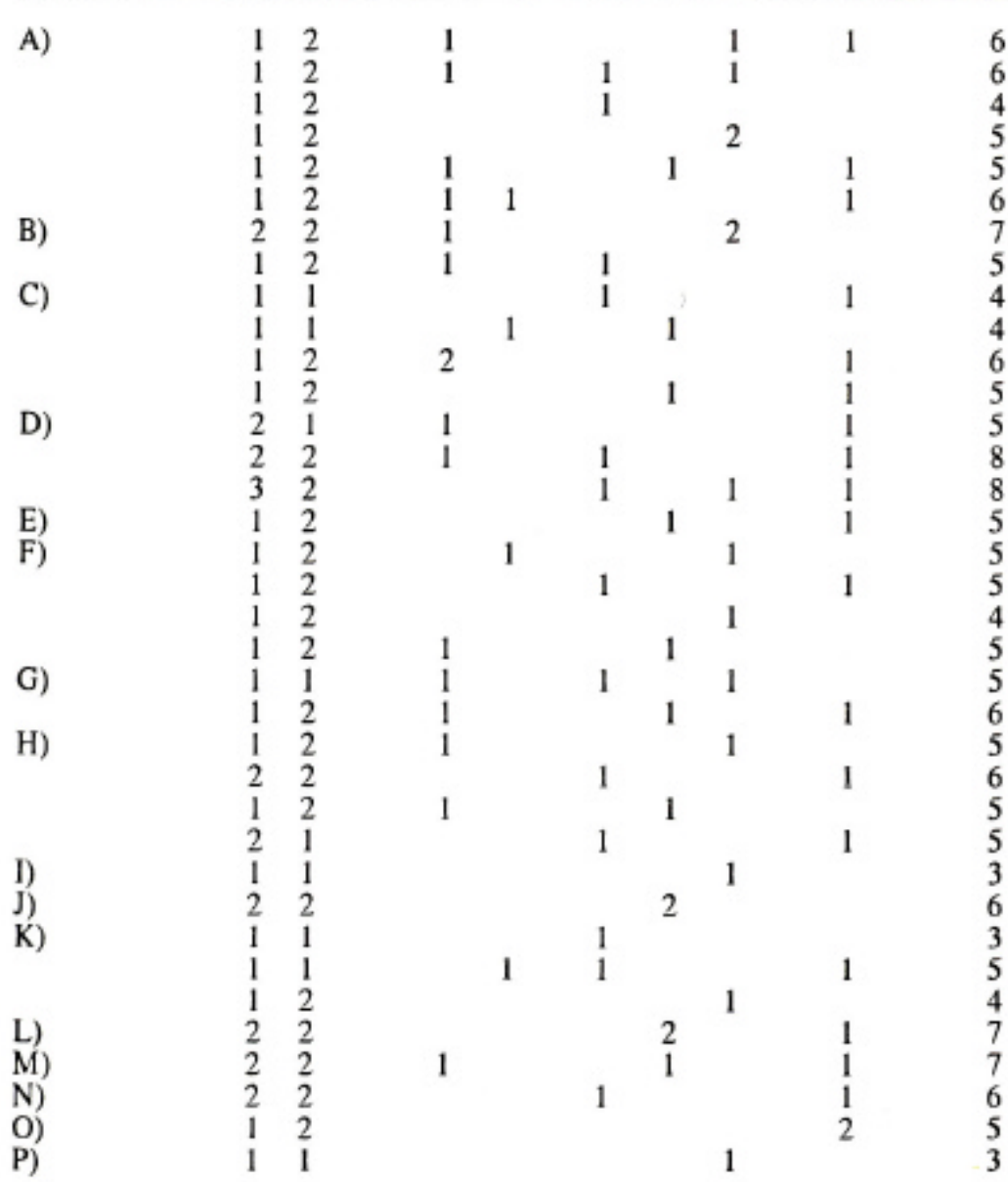

$\mathrm{M}=$ machos; $\mathrm{H}=$ hembras; $\mathrm{J} 2=$ juveniles mayores; $\mathrm{J} 1=$ juveniles menores; $\mathrm{J}=$ juveniles de edad indeterminada

- El período total de monitoreo de cada grupo varió de 1 a 6 años, por una muestra total, $\mathrm{N}=36$ grupo-años. 
TABLA 2. Densidad poblacional por número y biomasa de los primates diurnos de la Isla Chuana, Río Pacaya (F uente: Soini, 1986)

\begin{tabular}{|c|c|c|}
\hline Especie & $\begin{array}{l}\text { Densidad ecológica } \\
\text { (animales/ } \mathrm{K} \mathrm{m}^{2} \text { ) }\end{array}$ & $\begin{array}{l}\text { Biomasa* } \\
\left(\mathrm{Kg} / \mathrm{Km}^{2}\right)\end{array}$ \\
\hline Saguinus fuscicollis & 18.0 & 4.8 \\
\hline Saimiri boliviensis & 51.0 & 26.8 \\
\hline Cebus alfifrons & 4.2 & 7.2 \\
\hline Cebus apella & 8.7 & 16.3 \\
\hline Pithecia monachus & 12.8 & 14.4 \\
\hline A louatta seniculus & 36.0 & 162.0 \\
\hline Lagothrix lagotricha & 5.5 & 24.7 \\
\hline
\end{tabular}

Computo en base a los pesos empleados por Freese et at. (1982: Tabla VIII) en sus cómputos correspondientes. 
TABLA 3. Los recursos alimentarios de Alouatta seniculus en el Río Pacaya

\begin{tabular}{|c|c|c|c|c|c|c|c|c|c|c|c|c|c|c|}
\hline FAMILIA & ESPECIE & TIPO & $\mathbf{E}$ & $\mathrm{F}$ & M & $\mathrm{A}$ & $\mathbf{M}$ & $J$ & $J$ & A & s & $\mathrm{O}$ & $\mathbf{N}$ & $\mathrm{D}$ \\
\hline \multicolumn{15}{|l|}{ Recurse findos } \\
\hline ANACARDLACEAE & Spandias mombin & A & $\mathrm{x}$ & $x$ & & & & & & & & & & \\
\hline ANNONACEAE & Xylopia ligustrifolia & A & $\mathrm{x}$ & $\mathbf{x}$ & $\mathbf{x}$ & & & & & & & & & \\
\hline BOMBACACEAE & Ceiba pentandra & $A$ & & & & & & & $x$ & & & & & \\
\hline CONVOLVULACEAE & Muripa axilliflora & $\mathrm{L}$ & & & & & & & $\mathbf{x}$ & $\mathrm{x}$ & & & & \\
\hline FLACOURTIACEAE & Laetia corymbulase & $A$ & & & $x$ & & & & & & & & & \\
\hline \multirow[t]{3}{*}{ LEGUMGNOSAE } & Inga ingoides & A & $\mathrm{x}$ & & & & & & & & & & & \\
\hline & Inga marginaza & A & & $x$ & $\mathrm{x}$ & $\mathbf{x}$ & & & & & & & & $\mathbf{x}$ \\
\hline & Inga sp. & A & & $\mathbf{x}$ & & & $\mathbf{x}$ & & & & & & & \\
\hline MENISPERMACEAE & Abula freminum & L & $\mathbf{x}$ & & & $\mathbf{x}$ & & & & & $x$ & & & \\
\hline \multirow[t]{10}{*}{ MORACEAE } & Brosivum rubescens & $A$ & & & & $\mathrm{x}$ & $\mathbf{x}$ & & & & $x$ & & & \\
\hline & Cecropia membranaceae & A & $\mathrm{x}$ & $\mathrm{x}$ & $x$ & $\mathrm{x}$ & $x$ & $\mathbf{x}$ & & & & $\mathrm{x}$ & & \\
\hline & Clarisia bifora & A & $\mathbf{x}$ & $\mathrm{x}$ & $\mathrm{x}$ & $\mathrm{x}$ & $x$ & $\mathrm{x}$ & & $x$ & & $\mathbf{x}$ & $\mathrm{x}$ & $x$ \\
\hline & Coussapena sp. & $\mathbf{H}$ & & & & & & & & & & & $x$ & \\
\hline & Ficus manima & $\mathrm{H} / \mathrm{A}$ & $x$ & $x$ & $x$ & $x$ & $x$ & & & & & & $\mathbf{x}$ & \\
\hline & Finessp. A & $\mathrm{H} / \mathrm{A}$ & & & & & & & & & & $\mathrm{x}$ & $\mathbf{x}$ & \\
\hline & Ficus sp. B & A & & & & & & $x$ & & $\mathrm{x}$ & $\mathbf{x}$ & & & \\
\hline & Fleus ap. C & $\mathrm{H} / \mathrm{A}$ & & & & & & $\mathrm{x}$ & & & & & & \\
\hline & Ficus sp. D & H & $x$ & $\mathrm{x}$ & & & & $\mathbf{x}$ & & & & $\mathrm{x}$ & & \\
\hline & Ficus app. & $\mathrm{H} / \mathrm{A}$ & & $x$ & $\mathrm{x}$ & $x$ & $\mathbf{x}$ & & & $\mathrm{x}$ & $\mathrm{x}$ & $\mathrm{x}$ & & \\
\hline OLACACEAE & Cabtedra of. ocuminata & A & & $x$ & & & & & & & & & & \\
\hline \multirow[t]{2}{*}{ ROSACEAE } & Coucpia chrysoclayx & A & & & & & & & & & & & $\mathrm{x}$ & $\mathbf{x}$ \\
\hline & Licaria ajJ. apelala & A & & $\mathbf{x}$ & & & & & & & & & & $x$ \\
\hline SAPINDACEAE & Allophylins sp. & A & & & & & & & & & & & & $x$ \\
\hline SAPOTACEAE & Pontleria sp. & $A$ & $\mathrm{x}$ & & & & & & & & & & & \\
\hline \multicolumn{2}{|c|}{$\begin{array}{l}\text { Varias eapecies de árboles no ids. } \\
\text { Recurso hojas }\end{array}$} & & & & & $\mathrm{x}$ & & & & $\mathbf{x}$ & $x$ & $\mathbf{x}$ & & \\
\hline \multirow[t]{2}{*}{ BOMLIACACEAE } & Coiba peniandra & A & & & & & & & & $x$ & $x$ & $x$ & & \\
\hline & Pseudobowbiar mangunba & A & & & & & & & $\mathbf{x}$ & $x$ & & & & \\
\hline LECYTHIDACEAE & Couropila amazonica & A & & & & & & & & & & $\mathrm{x}$ & & \\
\hline \multirow[t]{6}{*}{ LEGUMINOSAE } & lnga marginata & A & $x$ & $\mathrm{x}$ & $\mathbf{x}$ & $x$ & $x$ & & & & & $\mathrm{x}$ & $\mathrm{x}$ & \\
\hline & liga punetata & A & & & & & $x$ & & & & & & & \\
\hline & linga sp. A & $A$ & & & & & & & $\mathbf{x}$ & & & & & \\
\hline & lnga sp. & A & $x$ & & $\mathbf{x}$ & $\mathrm{x}$ & $\mathrm{x}$ & & & & & & & \\
\hline & Macrobbium acacaefolium & A & & & & & $x$ & & & & & & & \\
\hline & Pinhecrolobium maltiflonum & A & $x$ & & $x$ & $x$ & $\mathbf{x}$ & $x$ & $\mathbf{x}$ & $\mathbf{x}$ & & $\mathbf{x}$ & $\mathbf{x}$ & \\
\hline MENISPERMACEAE & Abula fluminum & L & & $x$ & & & & & & & & & & \\
\hline \multirow[t]{3}{*}{ MORACEAE } & Cecropia membranaceae & A & $\mathrm{x}$ & $x$ & $x$ & $x$ & $\mathbf{x}$ & $\mathbf{x}$ & & $\mathrm{x}$ & $\mathbf{x}$ & $\mathrm{x}$ & $\mathbf{x}$ & $\mathrm{x}$ \\
\hline & Ficus sp. B & A & & & & & & $\mathbf{x}$ & $\mathbf{x}$ & $\mathrm{x}$ & & & & \\
\hline & Ficus spp. & $\mathrm{H} / \mathrm{A}$ & & $x$ & & $\mathbf{x}$ & & & & $\mathrm{x}$ & $\mathbf{x}$ & & $\mathrm{x}$ & \\
\hline $\begin{array}{l}\text { Varias especies no ids. } \\
\text { Recunso flores }\end{array}$ & & AyL & $\mathbf{x}$ & $x$ & $\mathbf{x}$ & $\mathbf{x}$ & $\mathrm{x}$ & $x$ & $x$ & $\mathrm{x}$ & $\mathrm{x}$ & $\mathrm{x}$ & $x$ & $\mathrm{x}$ \\
\hline ANNONACEAE & Xylopia ligustrifolia & A & & & & & & & & $\mathrm{x}$ & & & & \\
\hline APOCYNACEAE & Aspidasperma sp. & A & $x$ & & & & & & & & & & & \\
\hline BIGNONLACEAE & Paragonia pyriuidata & L & & & $x$ & & $x$ & & & & & & & \\
\hline BOMBACACEAE & Ceiba pentandra & $\bar{A}$ & & & & & & & $\mathrm{x}$ & & & & & \\
\hline \multirow[t]{3}{*}{ LEGUMINOSAE } & Inga punctata & A & & $\mathbf{x}$ & & & $x$ & & $\mathbf{x}$ & & & & & \\
\hline & Macrobbium actaciacfoliam & A & & & & & & & $x$ & & & & & \\
\hline & Plerocarpus amasanum & A & & & & & $x$ & & $\mathbf{x}$ & & & & & \\
\hline $\begin{array}{l}\text { Varias especies eo ids. } \\
\text { Recurso peciolos }\end{array}$ & & LyA & & $\mathrm{x}$ & $\mathbf{x}$ & $\mathrm{x}$ & $\mathbf{x}$ & $\mathrm{x}$ & & & & $\mathbf{x}$ & & \\
\hline \multirow[t]{2}{*}{ MORACEAE } & Cecropia membranacea & A & $x$ & & $\mathbf{x}$ & & & & & & & $\mathrm{x}$ & & \\
\hline & Fins sp. & A & & & & & & & $\mathrm{x}$ & & & & & \\
\hline
\end{tabular}

1 Tipo de plante : $\quad A=$ ábol; $H=$ hemiepifita; $L=$ Liana

Las aspas indican los meses ca que A. seniculus fue obervados alinentarse de la especie. 
TABLA 4. Composición estacional de la dieta alimentaria de Alouatta seniculus en el Río Pacaya medio, basada en relativa frecuencia de consumo observado de los diferentes recursos alimentarios.

\begin{tabular}{|l|l|l|l|l|}
\hline Recursos & $\begin{array}{c}\text { Estación Seca } \\
\text { (julio- set) }\end{array}$ & $\begin{array}{c}\text { Estación } \\
\text { inicial } \\
\text { de lluvia } \\
\text { (oct. dic) }\end{array}$ & $\begin{array}{c}\text { Estación } \\
\text { medio de } \\
\text { Iluvia } \\
\text { (ene- mar) }\end{array}$ & $\begin{array}{c}\text { Estación final } \\
\text { de lluvia } \\
\text { (abr- jun) }\end{array}$ \\
\hline Hojas & $64.0 \%$ & $61.5 \%$ & $30.1 \%$ & $45.5 \%$ \\
F rutos & $32.6 \%$ & $36.9 \%$ & $64.7 \%$ & $44.6 \%$ \\
Flores & $3.4 \%$ & $1.5 \%$ & $4.4 \%$ & $8.9 \%$ \\
Otros & - & $0.1 \%$ & $0.8 \%$ & $1.0 \%$ \\
\hline
\end{tabular}


FIGURA 1. El área de estudio Alouatta seniculus en Cahuana, Río Pacaya (^ Estación Biológica de Cahuana).

Límites del área principal de estudio. Los círculos numerados indican la distribución de todos los grupos heterosexuales de $A$. seniculus en la Isla Cahuana durante el censo poblacional. Escala aprox. 1:50,000.

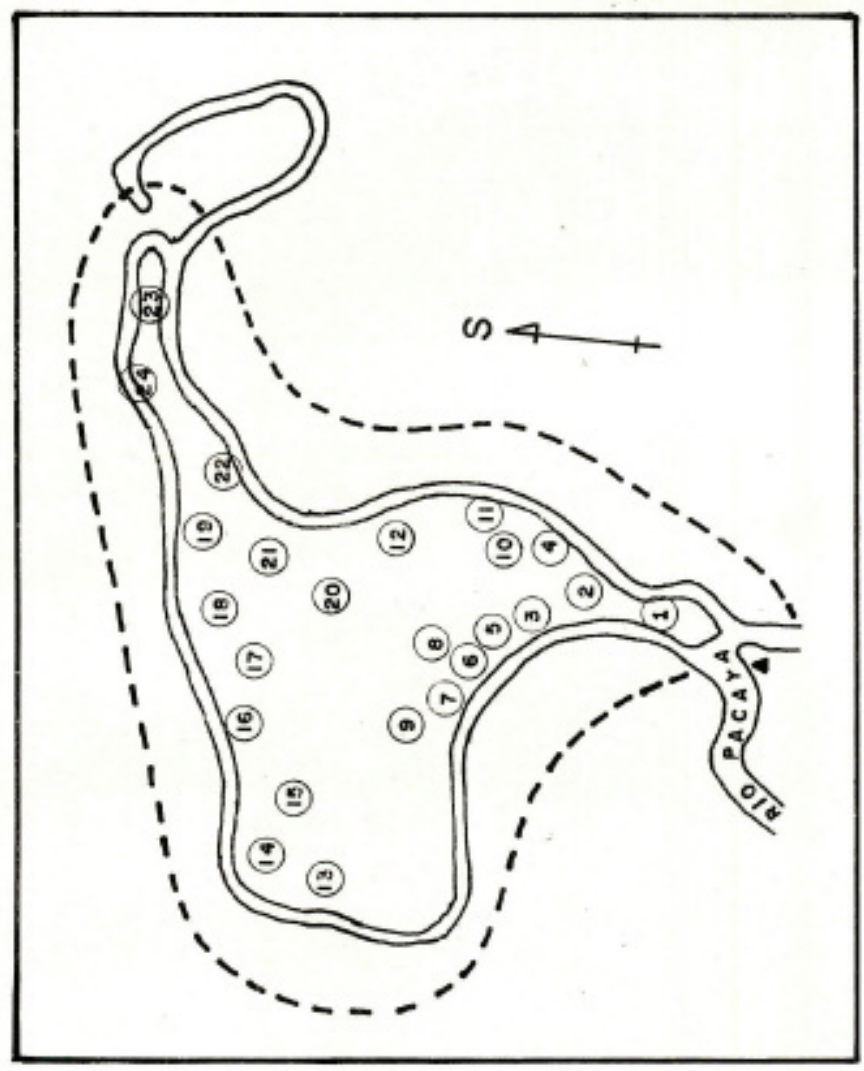


FIGURA 2. Promedios mensuales de precipitación y nivel del río en Cahuana, Río Pacaya en 1980-86.

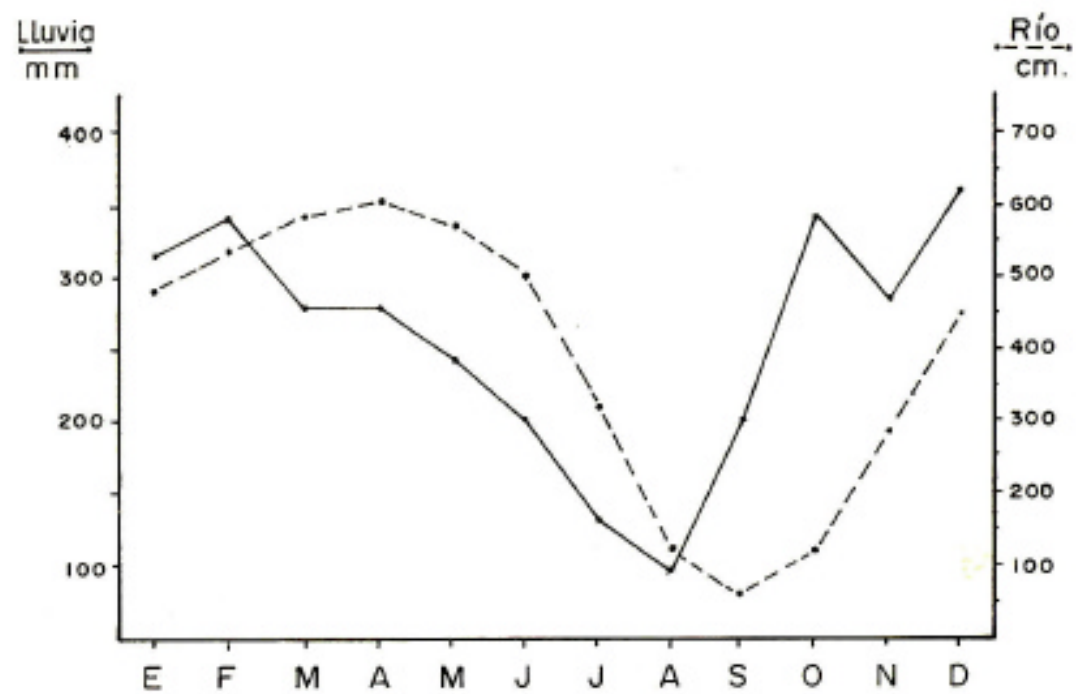


FIGURA 3. Distribución mensual de los nacimientos observados en la población de estudio de Alouatta seniculus

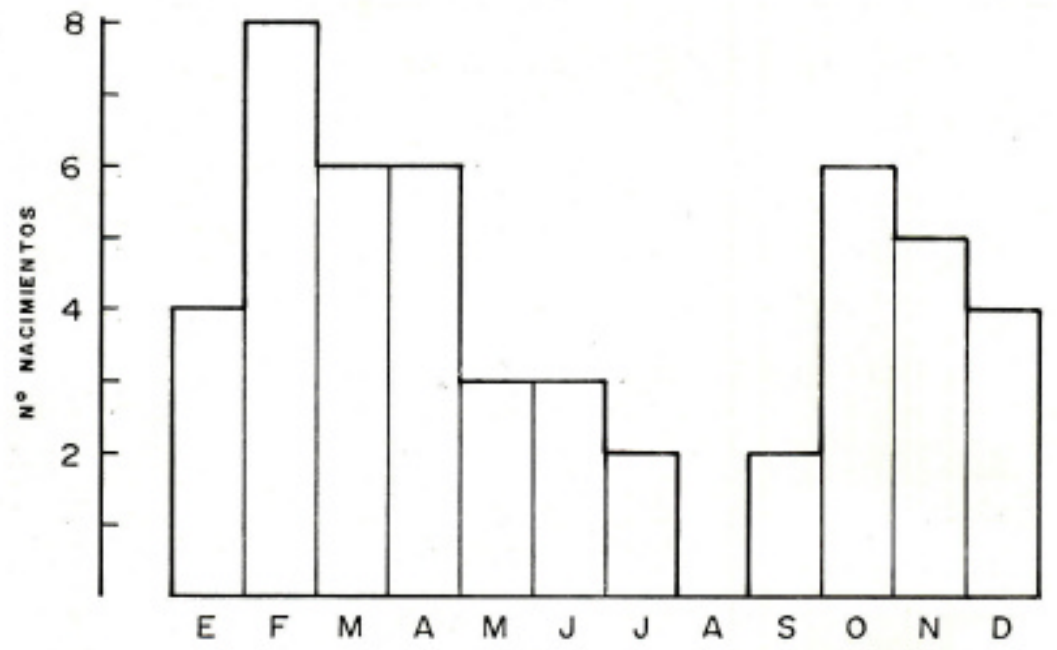

FIGURA 4. Las áreas domiciliarias de los grupos Nos. 1 y 2 de Alouatta seniculus en Isla Cahuana. Escala aprox. 1:12,500.

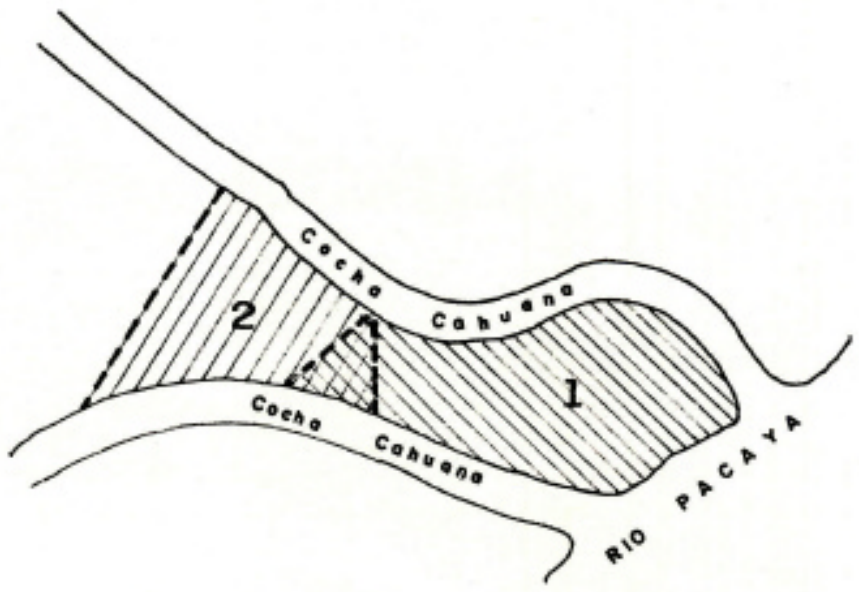

